\title{
Effect of the cluster angular momentum $J$ and the projectile orbital momentum $L$ on capture probability and postcollision dynamics
}

\author{
Massimo Mella ${ }^{\text {a) }}$ \\ School of Chemistry, Cardiff University, Cardiff CF10 3AT, United Kingdom
}

(Received 6 April 2009; accepted 9 September 2009; published online 30 September 2009)

In this work, collisions between rotating atomic clusters composed of Lennard-Jones $\left(\mathrm{LJ}_{n}\right)$ particles and an identical projectile have been investigated by means of trajectory simulations as a function of the cluster angular momentum $J$ and internal energy $E$, and for different values of the projectile impact parameter $b$ and relative velocity $v_{p}$. As expected, the collision $\left(P_{c}(b)\right)$ and capture [or sticking $\left.P_{s}(b)\right]$ probabilities are found to decay below unity for values of $b$ larger than the average surface radius of the cluster, with $d P / d b$ being strongly dependent on $v_{p}$. Both $P_{c}(b)$ and $P_{s}(b)$, however, appear to be largely insensitive to the modulus of the cluster angular momentum $|J|$ and only weakly dependent on $E$ for collisions involving target clusters with a lifetime $\tau>100$ ps. The latter findings are interpreted as indicating the absence of strong changes in the structure of the target as a function of $|J|$ and $E$. The comparison between the dissociation lifetime $\left(\tau_{\text {dyn }}\right)$ of the postcapture complexes $\left(\mathrm{LJ}_{n+1}^{*}\right)$ obtained continuing trajectories after monomer capture and the one computed from the fragmentation of statistically prepared clusters $\left(\tau_{\text {stat }}\right)$ supports the validity of a two-step capture-dissociation model; similarly, the comparison between the average amount of energy exchanged during trajectories $\left(\Delta E_{\text {dyn }}\right)$ in the process $\mathrm{LJ}_{n}+\mathrm{LJ} \rightarrow \mathrm{LJ}_{n+1}^{*} \rightarrow \mathrm{LJ}_{n}+\mathrm{LJ}$ and the one predicted by statistical simulations $\left(\Delta E_{\text {stat }}\right)$ suggests a fast statistical energy redistribution in the collisional complex even for very short $\tau_{\text {dyn }}$ (e.g., $40 \mathrm{ps}$ ). In the case of projectiles aimed at the edge of the cluster [(grazing collisions, $P_{c}(b)<1$ ]; however, the time elapsed between formal collision and dissociation, $\tau_{\text {coll }}$, is such that $\tau_{\text {coll }}<\tau_{\text {stat }}$ and the trajectories indicate the presence of ballistic dynamics and of a weak energy exchange $\left(\Delta E_{\text {coll }}<\Delta E_{\text {dyn }}\right.$, with $\Delta E_{\text {coll }}$ being the average energy exchanged during collisions). The relevance of these results to the study of gas phase nucleation and to the possibility of building a fully microcanonical framework for its description is discussed.

(C) 2009 American Institute of Physics. [doi:10.1063/1.3239476]

\section{INTRODUCTION}

The quest for an accurate description of a condensing vapor has recently led the interested community to embrace the use of dynamical and statistical simulations as a way to investigate the nucleation steps at atomistic level. ${ }^{1-7}$ Such an endeavor has recently highlighted several shortcomings of semiphenomemological theories such as classical nucleation theory, ${ }^{1,7}$ so that the necessity of introducing at least some extrathermodynamics (e.g., cluster chemical potentials) information is becoming apparent.

However, obtaining a fully "first principle" quantitive prediction for the nucleation flow $\mathcal{J}$ requires estimates for the forward $\left(k_{c}(n)\right)$ and backward $\left(k_{d}(n+1)\right)$ rates of the process $M_{n}+M \leftrightarrow M_{n+1}$ (Ref. 4) as a function of either temperature ${ }^{8}$ or energy ${ }^{9}$ to be available. As for the former variable, Schenter et $\mathrm{al}^{8}{ }^{8}$ have put forward a coherent framework, dynamical nucleation theory (DNT), based on transition state theory and classical canonical statistical simulations to investigate the time scale of nucleation in case of high background gas pressure. Whenever a microcanonical description ought instead to be preferred (e.g., at low collision frequency), ${ }^{9-11}$ the complexity of the underlying math-

${ }^{a)}$ Electronic mail: mellam@cardiff.ac.uk. ematical framework increases and it thus becomes important to exploit physically justifiable approximations as much as possible. ${ }^{10,11}$ For example, it is worth mentioning the previously highlighted apparent independence of $k_{d}(n+1)$ on the cluster angular momentum $J,{ }^{10,12,13}$ which may allow to neglect $J$ from the key variables needed to describe a condensing systems. Seeking for such and other possibilities, one may similarly wonder about the sensitivity of $k_{c}(n)$ on the angular momentum $J$ and energy $E$ of the target cluster, as well as if the energized complex $M_{n+1}^{*}$ formed right after a monomer capture event possesses properties largely independent of its formation process. As discussed in the following, whereas the trueness of the latter hypothesis would justify the coarse graining of time commonly employed in master equations (MEs) modeling, both $\partial k_{c} / \partial J \simeq 0$ and $\partial k_{c} / \partial E \simeq 0$ would allow one to substantially reduce the number of calculations needed to set up such system.

Somehow, the dependence of $k_{c}(n)$ on $J$ and $E$ does not seem of having been sufficiently investigated to satisfactorily answer such questions even in the simple case of LennardJones (LJ) atoms $\left(\mathrm{LJ}_{n}+\mathrm{LJ} \rightarrow \mathrm{LJ}_{n+1}\right){ }^{14-16}$ Given our interest in developing a microcanonical version of DNT, investigating the sensitivity of $k_{c}(n)$ with respect to $J$ and $E$ is thus highly important and represents our main goal in this work. 
We tackle such task using trajectory simulations to compute both $P_{c}(n, b, E, J)$ (Ref. 16) and $P_{s}(n, b, E, J)$, respectively, the probability of collision and capture (or sticking, vide infra for definitions) between a $\mathrm{LJ}_{n}$ cluster with internal energy $E$ and angular momentum $J$, and a monomer with impact parameter $b$. With the total collision or capture rates for a chosen set of condition $n, E, J$, and relative projectile velocity $v_{p}$ being given by the equation $2 \pi v_{p} \int_{o}^{\infty} P(n, b, E, J) b d b$, the differential quantities $P_{c}$ and $P_{s}$ provide one with all the needed information on the behavior of $k_{c}(n)$.

Additionally, a detailed test of the accuracy of decomposing monomer-cluster collisional dynamics in two physically meaningful steps (capture and dissociation) and how this is affected by $J$ and $E$ is only partially available from previous works. This is mainly due to the choice of using only cold clusters (i.e., $E=0)($ Ref. 15) or the lack of a direct comparison with appropriate data. ${ }^{17}$ To improve on the current situation, we have selected to compare the average lifetime $\left(\tau_{\text {dyn }}\right)$, as well as its distribution, of collisionally formed $M_{n+1}^{*}$ clusters with the one $\left(\tau_{\text {stat }}\right)$ of microcanonically ${ }^{18}$ prepared counterparts (i.e., with identical $E$ and $J$ ) for noncold species. Additionally, we compared the average collisional energy transfer as a function of the impact parameter $b$ obtained as direct results of trajectory simulations $(\Delta E(b))$ with the prediction made by a two step statistical procedure $\left(\Delta E_{\text {stat }}(b)\right)$. Since in the latter we assumed the existence and equilibrium nature of $M_{n+1}^{*}$, such comparison would help in validating the idea that $M_{n+1}^{*}$ intermediates have properties largely independent of the details of their formation by quantifying the possible effects of postcollisional nonstatistical behavior (e.g., nonstatistical dissociation branching ratios). We consider such investigation as the second goal of our work.

The rest of this manuscript is organized as follows. In Sec. II, the methodology of this study is presented. Section III reports and discusses the numerical results. Finally, Sec. IV contains our conclusions and avenues for future investigations.

\section{THEORETICAL METHODS}

LJ particles interact by means of a sum of pair potentials of the form $\mathcal{V}=\sum_{i j} V_{\mathrm{LJ}}\left(r_{i j}\right)=\sum_{i j} 4 \epsilon\left[\left(\sigma / r_{i j}\right)^{12}-\left(\sigma / r_{i j}\right)^{6}\right]$, where $\epsilon=3.7935 \times 10^{-4}$ hartree $(0.23805 \mathrm{kcal} / \mathrm{mol})$, $\sigma=6.4354$ bohr $(3.4050 \AA)$, and the atomic mass $m$ $=73350.6$ times the electron mass were used to roughly represent Ar aggregates in our calculations.

All cluster sizes were equilibrated at the chosen values of internal (vibrational plus rotational) energy $E$ by means of the efficient microcanonical sampling (EMS) procedure $^{18}$ employing, at least, $10^{6}$ Monte Carlo steps per particle before initiating trajectory simulations. To avoid evaporation during the Monte Carlo sampling, a constrain was placed on the maximum distance between a particle and the cluster center of mass $(\mathrm{CoM})\left(r_{\mathrm{CoM}}\right)$ such that $r_{\mathrm{CoM}}<6 \sigma$; the latter was enforced rejecting displacements leading to $r_{\mathrm{CoM}}>6 \sigma$.

Estimates for the lifetime $\tau_{\text {stat }}$ of clusters in the statistical NVE ensemble were obtained by means of trajectory simulations, with initial particle positions $\mathbf{R}=\left(\mathbf{r}_{1}, \mathbf{r}_{2}, \ldots, \mathbf{r}_{n}\right)$ being generated using EMS and initial momenta $\mathbf{P}$ $=\left(\mathbf{p}_{1}, \mathbf{p}_{2}, \ldots, \mathbf{p}_{n}\right)$ being randomly sampled as described previously $^{18}$ and fulfilling the condition $\sum_{i=1}^{n} \mathbf{p}_{i}^{2} / 2 m=E$ $-\mathcal{V}(\mathbf{R})$. Notice that the adjective "statistical" refers only to the choice of trajectory initial conditions in this context, and not to system behavior or to the use of a statistical theory to estimate $\tau_{\text {stat }}$. Given the very weak sensitivity of $\tau_{\text {stat }}$ with respect to the total angular momentum $J$ of the fragmenting cluster highlighted previously, ${ }^{10,12,13}$ no restriction was imposed on the possible value of $J$, while computing it. The latter was obtained using at least 2500 trajectories for each energy $E$, with the equation of motion having been integrated with the leap-frog algorithm for up to 120 ps unless dissociated. After some initial experiments testing for a possible dependency of $\tau_{\text {stat }}$ on the definition of "dissociation," it was found appropriate to dub a trajectory as dissociated when $r_{\mathrm{CoM}}>6 \sigma$ for any LJ particle. A time step of 5 fs was used in the trajectory integration, which led to an energy conservation better that $100 \mathrm{ppm}$ in every case. An estimate for $\tau$ was obtained fitting the long time behavior of $\ln \left[\left(N_{0}-N_{t}\right) / N_{0}\right]$ with a straight line of the form $a+t / \tau_{\text {stat }}$. Here, $N_{0}$ and $N_{t}$ are, respectively, the total number of trajectories used in the simulation and the number of dissociated trajectories at time $t$. Notice that the choice of fitting only the long time behavior is made in order to eliminate possible transient due to slow intramolecular energy distribution (i.e., intrinsically nonRice-Ramsberger-Kassel-Marcus dissociation ${ }^{19}$ ). However, cluster dissociation appeared to closely follow an exponential behavior (vide infra) in the range of energy explored.

Collision $\left(P_{c}(n, b, E, J)\right)$ and capture $\left(P_{s}(n, b, E, J)\right)$ probabilities between a $\mathrm{LJ}_{n}$ cluster and a monomer are defined as the fraction of trajectories $\left(N_{c} / N_{0}\right.$ or $\left.N_{s} / N_{0}\right)$ that led to a monomer-cluster collision or capture for a chosen set of ensemble conditions (i.e., $n, b, E, J$, and $v_{p}$ ). A collision was deemed as happened when the distance between the projectile and any of the cluster particles was less than a chosen threshold $r_{c}$. In other words, the distance-based Stillinger definition for a cluster ${ }^{20}$ was used to monitor for a collision. Since quantities such as $P_{c}(n, b, E, J)$ may be sensitive to the Stillinger distance $r_{c}$ that defines interatomic connectivity, we used $r_{c}=(2)^{1 / 6} \sigma$, the equilibrium distance for $\mathrm{LJ}_{2}$, to guarantee the presence of a strong interaction between projectile and cluster particles. Conversely, a capture event was deemed as happened after a collision when the projection of the relative projectile-cluster velocity $v_{p}$ along the vector joining the CoM of the two species changed sign a chosen number of times $N_{\mathrm{tp}}$. As before, ${ }^{21}$ we found $P_{s}(n, b, E, J)$ to be very weakly dependent on $N_{\text {tp }}$ and $N_{\text {tp }}=3$ was employed as definition.

To compute $P_{c}(n, b, E, J)$ and $P_{s}(n, b, E, J)$, the projectile was initially placed randomly on a sphere with radius $r_{i}=6 \sigma$ centered on the CoM of the target cluster. At this distance, the interaction between the impinging monomer and the cluster is negligible, so that the cluster angular momentum $J$ and the orbital angular momentum $L$ of the two fragments are well defined. Initial positions and momenta for the particles in the target cluster were chosen as described above after equilibration with EMS at the appropriate internal energy $E$ using at least $10^{6}$ Monte Carlo steps per par- 
ticle. $J$-selected initial condition were obtained by simply rejecting initial momenta that did not satisfy $\left|J-J_{\text {ini }}\right| \leq 3$, where $J_{\text {ini }}$ is the cluster angular momentum computed with that particular choice of momenta. The largest value for the cluster angular momentum $|J|_{\max }$ explored in the simulations for each $\mathrm{LJ}_{n}$ was such that $\int_{0}^{|J| \max } p(|J|, E) d|J| / \int_{0}^{\infty} p(|J|, E) d|J|$ $>0.90$, where $p(|J|, E)$ is the probability distribution for $|J|$ in the microcanonical ensemble at the chosen $E$ as obtained from $N V E$ simulations similar to the ones used to estimate $\tau_{\text {stat. }}$. In all cases, $p(|J|, E)$ was well approximated by a canonical-like density $p(J, E) \sim J^{2} \exp \left[-\alpha(E) J^{2}\right]$, perhaps suggesting that the number of states for $3 n-6$ internal degrees of freedom of $\mathrm{LJ}_{8,14,19}$ is sufficiently large to simulate the behavior of a heat bath with respect to the 3 rotational degrees of freedom. The direction of the initial relative velocity $v_{p}$ between projectile and cluster was selected randomly among the ones suitable for the chosen impact parameter $b$. The two different values $v_{p}=0.0001$ and 0.0002 a.u., respectively, corresponding to a relative kinetic energy $\epsilon_{p}$ $=m v_{p}^{2} / 2=0.23$ and $0.92 \mathrm{kcal} / \mathrm{mol}$, were used to investigate the dependency of $P_{c}$ and $P_{s}$ on the monomer collision speed. The lowest speed was chosen in order to represent the most likely velocity for $\mathrm{Ar}$ at $120 \mathrm{~K}\left(v_{p}=0.0001\right.$ a.u. $)$, whereas the higher was chosen to provide information on high speed collisions despite they represent only infrequent events (the relative probability for the two velocities is $\sim 0.2$ at $120 \mathrm{~K})$. Compared with previous published work, ${ }^{14,16,17}$ the two chosen values of $v_{p}$ allow one a more thorough investigation of the previously largely omitted high energy collision range, for which one may expect the presence of interesting nonstatistical effects especially at large $b$ values.

Trajectory simulations were used to investigate only the range of $b$ around which $P_{s}$ and $P_{s}$ are rapidly varying, as obtained from a preliminary exploration over a wider set of values. Equation of motions was integrated as described above up to a total postcollision evolution time of $1000 \mathrm{ps}$. For every specific choice of the three parameters $(b, E, J)$, 1000 trajectories were run to accumulate adequate statistics. Postcollision $\left(\tau_{\text {coll }}\right)$ and capture $\left(\tau_{\text {dyn }}\right)$ lifetimes, which differ due to possible "nonsticky" $\left(N_{\mathrm{tp}}<3\right)$ collisions, were extracted using the same procedure employed for microcanonically prepared dissociating clusters using as time origin the collision event. In other words, after a collision has happened $\left(r_{c}<(2)^{1 / 6} \sigma\right)$, trajectories are followed until a particle detaches from $M_{n+1}^{*}$ (i.e., $r_{\mathrm{CoM}}>6 \sigma$ ), with the time elapsed between collision and dissociation being recorded and labeled as "postcollision" or "postcapture" lifetime according to our definitions. A linear fit of the long time behavior of $\ln \left[\left(N_{0}-N_{t}\right) / N_{0}\right]$, computed using the recorded time lags for the two ensembles of trajectories, provides an estimate for $\tau_{\text {coll }}$ and $\tau_{\text {dyn }}$. As in the calculation of $\tau_{\text {stat }}$, the distribution of both $\tau_{\text {coll }}$ and $\tau_{\text {dyn }}$ was found to closely follow an exponential behavior (vide infra).

\section{RESULTS AND DISCUSSION}

The possible dependency of $P_{c}$ and $P_{s}$ on $n$ was explored studying the three cluster sizes $\left(\mathrm{LJ}_{8,14,19}\right)$ previously employed. ${ }^{10}$ The internal energy of these target clusters was
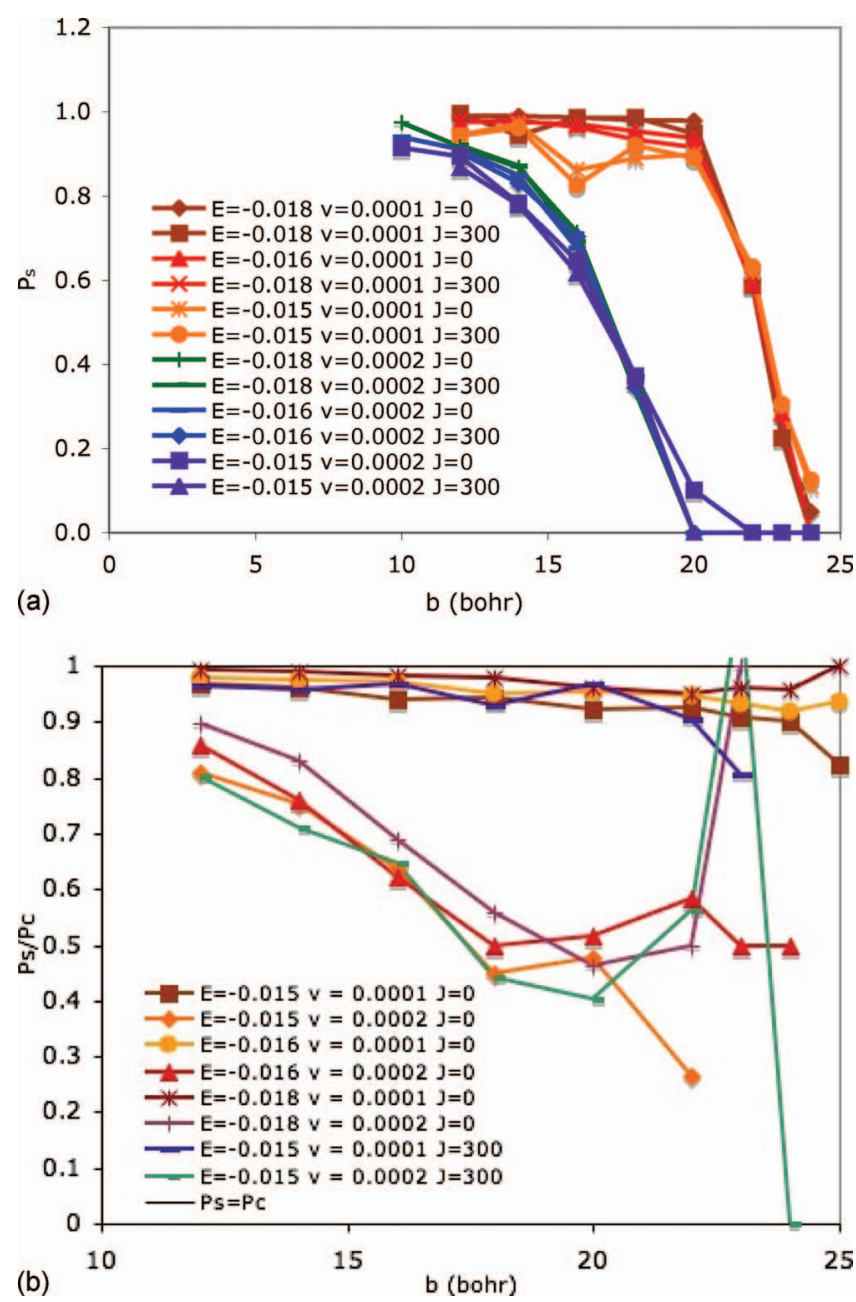

FIG. 1. (a) (Top) Behavior of the monomer collision probability $P_{c}$ as a function of the impact parameter $b$ for $\mathrm{LJ}_{19}$. The graphs show twelve $\left(E, v_{p}, J\right)$ cases, with $E=-0.018,-0.016$, and -0.015 hartree (corresponding to and internal energy of $0.00956,0.01156$, and 0.01256 hartree or $25.2,30.5$, and $33.1 \mathrm{kcal} / \mathrm{mol}), \quad J=0$ and $J=J_{\max }=300 \hbar$, and $v_{p}$ $=0.0001,0.0002$ a.u. (equivalent to a kinetic energy of 0.23 and $0.92 \mathrm{kcal} /$ mol). Statistical errors are of the order of 3\%-4\% of the shown values; (b) (bottom) behavior of $P_{s} / P_{c}$ as a function of the impact parameter $b$ for $\mathrm{LJ}_{19}$. The graphs show six $\left(E, v_{p}, J\right)$ cases, with $E=-0.018,-0.016$, and -0.015 hartree (corresponding to an internal energy of $0.00956,0.01156$, and 0.01256 hartree or $25.2,30.5$, and $33.1 \mathrm{kcal} / \mathrm{mol}), J=0 \hbar$, and $v_{p}$ $=0.0001,0.0002$ a.u. (equivalent to a kinetic energy of 0.23 and $0.92 \mathrm{kcal} /$ mol). Also shown are the cases: $E=-0.015$ hartree, $J=300 \hbar$, and $v_{p}$ $=0.0001,0.0002$ a.u.. Statistical errors are of the order of $3 \%-4 \%$ of the shown values, apart when $b>18$ and $v_{p}=0.0002$ a.u. where they are of the same order of magnitude due to the small number of collisions.

such that their dissociation lifetime $\tau_{\mathrm{TST}}$, as predicted using transition state theory, was in the range $100 \leq \tau_{\text {TST }}$ $\leq 1000 \mathrm{ps} ;{ }^{10}$ depending on the energy, the aggregates are either liquidlike or solidlike with fluxional surfaces. Given the qualitatively similar behavior for all sizes, we describe only results for $\mathrm{LJ}_{19}$; however, some comparative data for $\mathrm{LJ}_{8}$ and $\mathrm{LJ}_{14}$ (mainly $P_{c}$ and $\tau_{\text {coll }}$ ) are provided in the supporting information (Tables I-XXVI). ${ }^{22}$

Panel a of Fig. 1 shows $P_{c}(19, b, E, J)$ as a function of $b$, $E$ for $J=0$, and $J=J_{\max }$, and $v_{p}=0.0001,0.0002$ a.u. According to its definition, $P_{c}$ provides indications on the geometrical size of the target cluster as well as on its capability of deviating the projectile from its initial trajectory. As seen, 


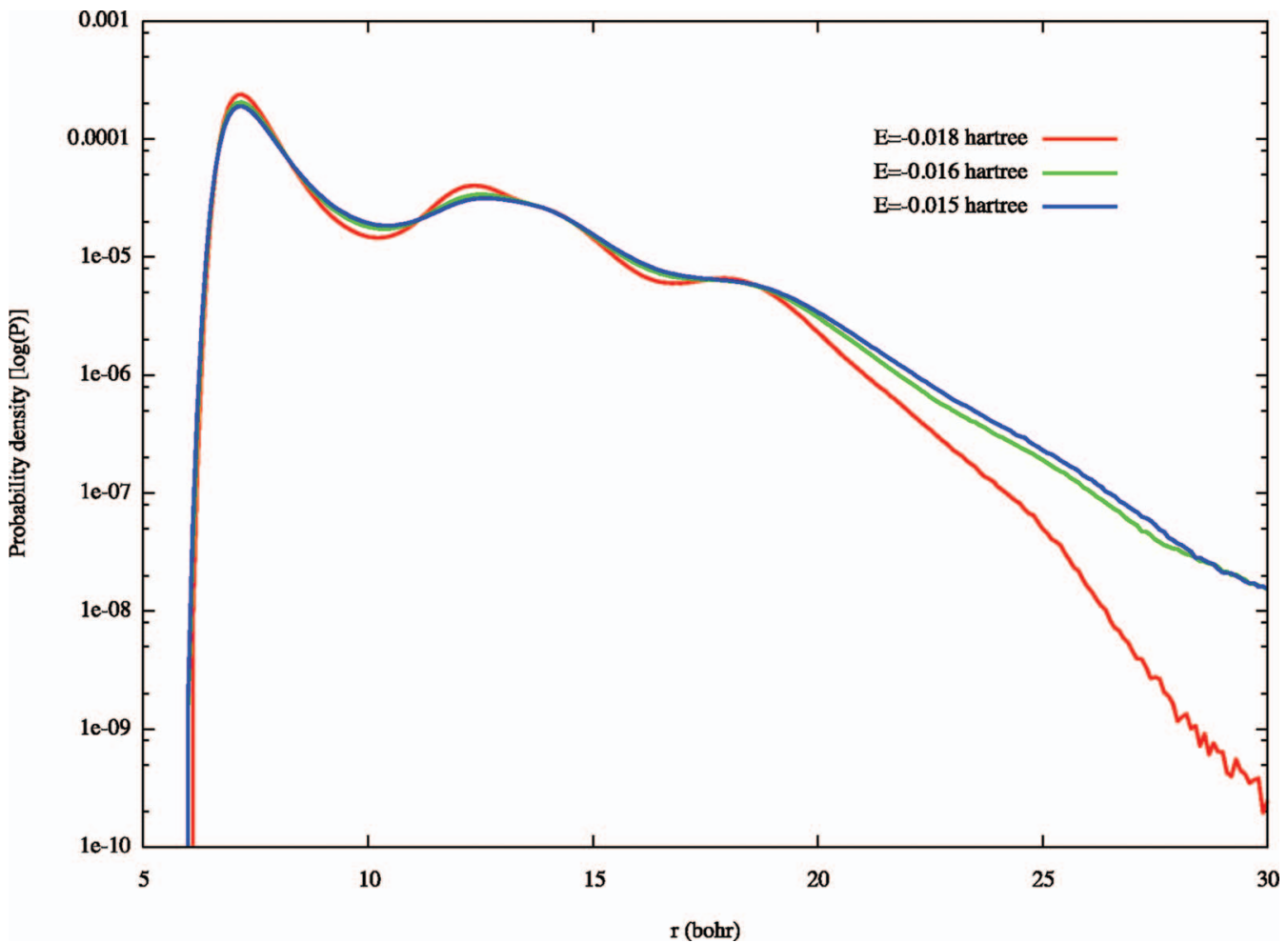

FIG. 2. Probability distribution function $P(r)$ for the distance $r$ between a $\mathrm{LJ}$ particle and the $\mathrm{CoM}$ of $\mathrm{LJ}_{19}$. $P(r)$, normalized so that $\int P(r) r^{2} d r=1$, is represented in logarithmic scale. Distances in bohr.

$P_{c}(n, b, E, J)$ remains close to unity until $b$ is such that projectiles are fired close to the cluster edge and falls sharply at longer distances from the CoM, in agreement with earlier work. ${ }^{14-16}$ Similarly, $P_{c}(n, b, E, J)$ decays away from unity at values of $b$ and with a slope that depend on the projectile velocity $v_{p}$ (higher $v_{p}$ being associated with lower $b$ and reduced slopes). ${ }^{14,15}$ A close inspection of Fig. 1(a) also shows that $P_{c}(n, b, E, J)$ weakly increases/decreases at large/ small value of $b$ upon increasing $E$; this effect can be attributed to an increase in cluster volume upon increasing its total energy content as shown in Fig. 2. This effect is however marginal at low $v_{p}$ for the range of energies explored in this work, whereas it may fractionally change the total collision probability at higher projectile speeds. The more interesting observation that can be extracted from Fig. 1 is that $P_{c}(n, b, E, J)$ appears to be largely independent of $J$ over the explored range, a finding reflecting a similar lack of structural changes due to the low energy content of the rotational motion. As an additional confirmation of this finding, trajectory simulations have been carried out without selection of the initial value of $J$ for the target clusters with the additional results fully agreeing with the $J$-selected ones.

Panel b of Fig. 1 shows the ratio $P_{s} / P_{c}$ as a function of $b$ for the case $J=0 \hbar$, which provides information on the postcollision capture capability of the cluster. This quantity can also be used to quantify the propensity of the target cluster to act as energy sink for the projectile and its dependency on the impact parameter; in fact, without energy exchange between the two species no capture would happen. Selected $J$ $=300 \hbar$ cases highlight that no dependency is present for $P_{s} / P_{c}$ on $J$ over the range explored, thus indicating once again the minor role played by this quantity on the descrip- tion of collision/capture events in atomic clusters. From this figure it also emerges that the behavior of $P_{s} / P_{c}$ is dependent on $v_{p}$. At low $v_{p}$, more than $90 \%$ of collision produce a capture event, the actual quantitative values showing only a small changes over the range of $b$ and energy explored. In the same condition, we notice that $P_{s} / P_{c}$ decreases mildly upon increasing $b$ and $E$, indicating a slightly more effective energy exchange between projectile and target when the cluster is "cold" and the collision more direct. In the case of higher $v_{p}$, instead, the dependency of $P_{s} / P_{c}$ on $b$ intensifies, with values well below unity indicating the reduced capability of the target to deviate the projectile from its initial trajectory and to incorporate it, at least temporarily.

Given the definition of collision adopted in this study $\left(r_{c} \leq 2^{1 / 6} \sigma\right)$, it seems likely that the large differences between $P_{s}$ and $P_{c}$ at large speed could be ascribed primarily to nonballistic dynamics, i.e., to the conservation of a fraction of the tangent component of the projectile velocity $\left(v_{p}\right)$ upon collision. Whether this is or not the case, it can be investigated by looking at the branching ratio for the monomer detachment after projectile collision or capture. Figure 3(a) shows the fraction of each isomer produced (labeled according to which monomer has abandoned the energized $M_{n+1}^{*}$ ) following a simple collision (i.e., without capture) between $\mathrm{LJ}_{19}$ and a $\mathrm{LJ}$ monomer at the lowest of the three energies and both $v_{p}$ values. From panel a of Fig. 3, it is clearly seen that the projectile leaves more frequently after a collisional event than any other particles the higher $v_{p}$ and $b$ are; in fact, the high $v_{p} /$ high $b$ case is dominated by the projectile leaving. A similar situation is present also when $b=14$ bohr and $v_{p}=0.0002$ a.u., where, however, the almost unity collision probability suggests an almost elastic rebound to be the 

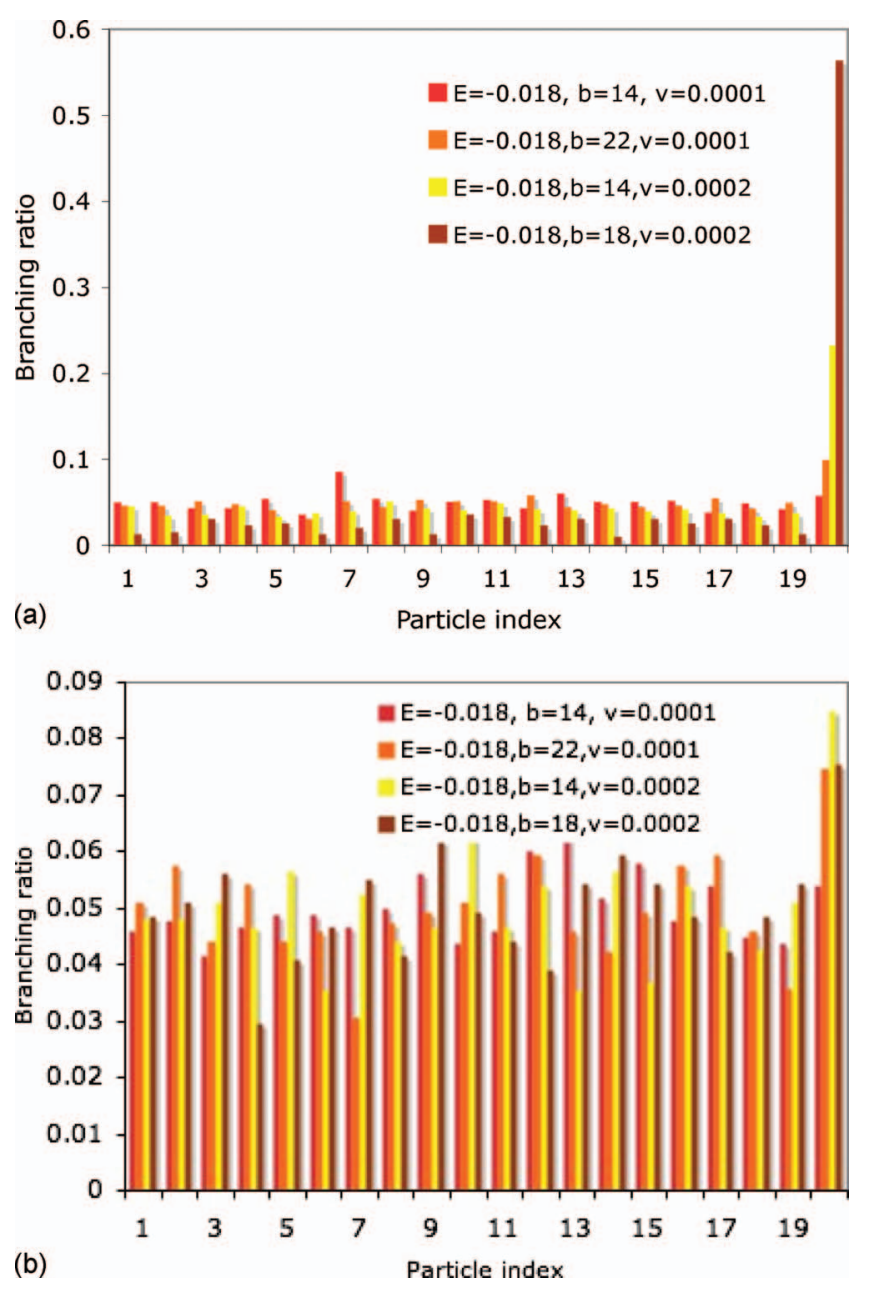

FIG. 3. Branching ratio (fractional number of each isomer dissociated) for the postcollisional (panel a, top) and postcapture (panel b, bottom) fragmentation of $\mathrm{LJ}_{20}^{*}$; results obtained using 1000 trajectories at the indicated values of $E, b$, and $v_{p}$ (in atomic units, see caption Fig. 1 for conversion). Notice the decrease in the branching ratio for the projectile (particle 20) upon decreasing the value of $b$ and $v_{p}$ present in panel a. The statistical branching ratio is 0.05 .

source of such behavior. At variance with the latter cases, the branching ratio at low $v_{p}$ behaves essentially in a statistical way despite the difference in impact parameter.

Similarly, the branching ratio when the projectile is captured by the target (panel b, Fig. 3) is clearly consistent (within the fluctuations in different channels) with a statistical behavior and largely independent on the trajectory details. In other words, whenever captured, even the nature of a fast projectile is quickly "randomized" by the coupling with the internal mode of the target cluster. Because the ratio $P_{s} / P_{c}$ appears independent of $J$, this conclusion appears to be valid irrespective of the angular momentum of the target, thus giving support to the possibility that microcanonical (but not $J$-resolved) statistical theories may be able to provide an adequate description of nucleating species ${ }^{10,17}$ (vide infra for a more quantitative discussion).

Support to the notion just discussed stems also from the lack of sensitivity with respect to $J$ and $b$ found for the lifetime $\tau_{\text {dyn }}$ of the postcapture $\mathrm{LJ}_{n+1}^{*}$ (Fig. 4, panel a) that, apart from the wide fluctuations present when $P_{s}$ is very low, is in good agreement with and extends our previous results ${ }^{10}$
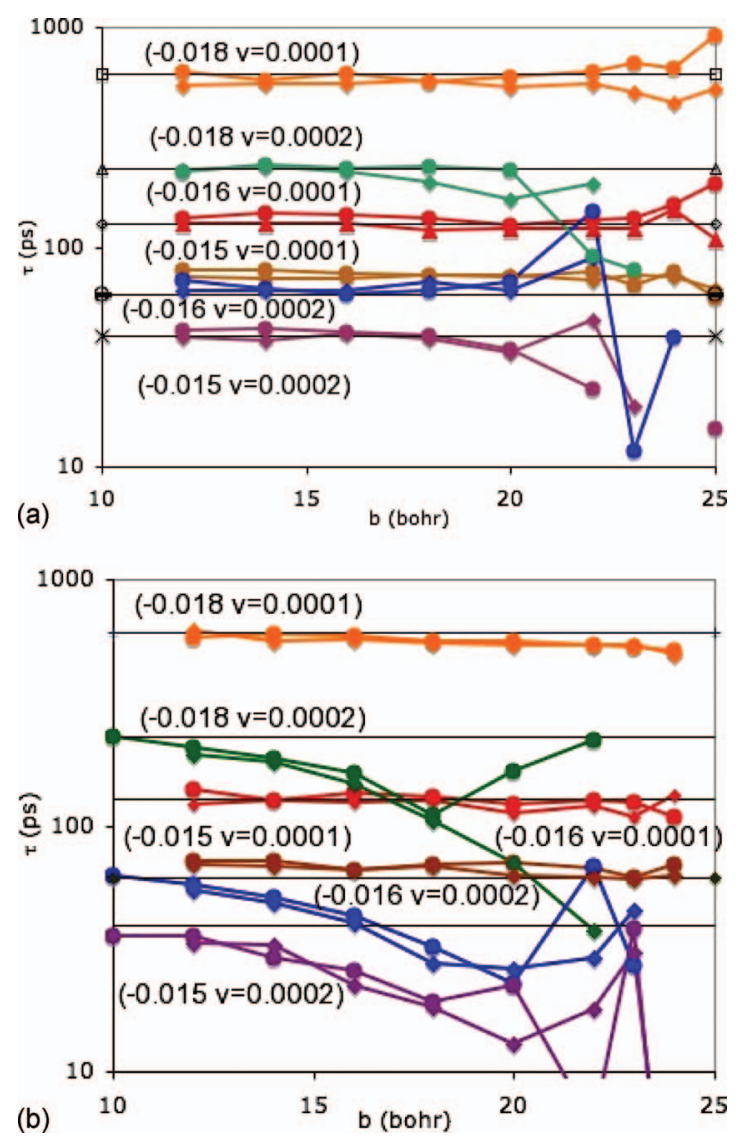

FIG. 4. Dependency of the $\mathrm{LJ}_{20}^{*}$ postcapture $\tau_{\text {dyn }}$ (panel a, top) and postcollisional $\tau_{\text {coll }}$ (panel $\mathrm{b}$, bottom) lifetime on the impact parameter $b$. The graphs show twelve $\left(E, v_{p}, J\right)$ cases, with $E=-0.018,-0.016$, and -0.015 hartree, $J=0$ (circle) and $J=J_{\max }=300$ (diamonds) a.u., and $v_{p}$ $=0.0001,0.0002$ a.u. (see caption Fig. 1 for conversion). The horizontal lines show the lifetime $\tau_{\text {stat }}$ for clusters statistically prepared with the same internal energy $E$ of collisional complexes. Statistical errors are of the order of $3 \%-4 \%$ of the shown values for $b \leq 18$ bohr, while they have the same order of magnitude of the shown data due to the much lower collision probability for $b \geq 18$ bohr.

to clusters with higher total angular momentum up to $J^{\prime}=J$ $+m\left|b \times v_{p}\right|$ ( $m$ is the mass of the projectile). Moreover, $\tau_{\mathrm{dyn}}$, which is computed by analyzing the postcapture decay of energized $M_{n+1}^{*}$ species, closely agrees with the lifetime $\tau_{\text {stat }}$, the one obtained using trajectories starting from statistically prepared initial conditions; such an agreement indicates that the absence of nonstatistical branching ratios shown in Fig. 3 (b) is reflected in statistically identical decaying times as a result of fast intramolecular vibrational energy redistribution. An even more thorough check of this idea is given by the lifetime distribution $P(t)$ of $M_{n+1}^{*}$, which provides a much more sensitive quantity to the presence of nonstatistical behavior than the average lifetime. Panel a of Fig. 5 shows the $P(t)$ obtained for $E=-0.018$ hartree, $v_{p}=0.0001$ and 0.0002 a.u., and two different values of impact parameter chosen to represent situation of high $(b=14$ bohr $)$ and low ( $b$ $=22$ bohr at low $v_{p}$ and $b=18$ bohr at high $v_{p}$ ) sticking probabilities. As expected, the data indicate a faster decays following high speed collisions due to a higher internal energy of $\mathrm{LJ}_{n+1}^{*}$ but also the fact that, within statistical fluctuations, the impact parameter does not play an important role in defining the quantitative features of $P(t)$ whatever the ini- 

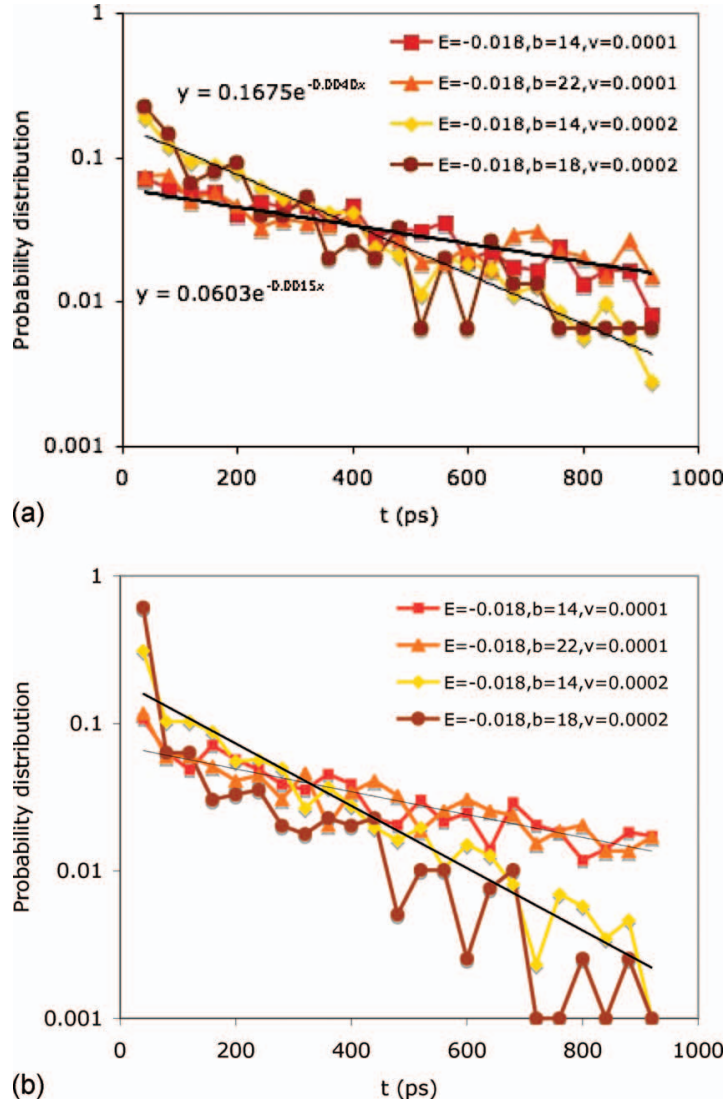

FIG. 5. Probability distribution function $P(\tau)$ for the postcapture $\left(\tau_{\text {dyn }}\right.$, panel a) and postcollisional $\left(\tau_{\text {coll }}\right.$, panel b) lifetimes of $\mathrm{LJ}_{20}^{*}$; results obtained using 1000 trajectories at the indicated values of $E, b$, and $v_{p}$ (in atomic units, see caption Fig. 1 for conversion) with a coarse graining time interval of $\Delta \tau$ $=40$ ps for the distribution. $P(\tau)$, normalized so that $\int P(\tau) d \tau=1$, is represented in logarithmic scale. Thin and thick black lines are fitted to the long time behavior of $P(\tau)$ at $\left(b=22, v_{p}=0.0001\right)$ and $\left(b=14, v_{p}=0.0002\right)$. In panel $\mathrm{b}$, notice the high value of $P(40)$ for $v_{p}=0.0002$, mostly due to the fast dissociation of the projectile from the target, and the fact that it decrease upon decreasing $b$. Also, notice the similar decay of $\mathrm{LJ}_{20}^{*}$ at long survival time for species with the identical internal energy, indicating the presence of a statistical lifetime distribution.

tial value of $v_{p}$. Also, the average decay time $T$ extracted from fitting the lifetime distributions with an analytical function of the form $P(t)=T^{-1} \exp [-t / T]$, respectively, $\sim 250$ and $\sim 660$ ps at high and low $v_{p}$, agrees to better than $10 \%$ with $\tau_{\text {stat }}$ and $\tau_{\text {dyn }}$.

The findings described above are somewhat at variance with the results for the postcollision lifetime distributions (i.e., including all clusters for which a collision as happened) in panel b of Fig. 5, which clearly show a nonstatistical distribution of lifetimes in the range $0<t \leq 40$ ps at high $v_{p}$, and with the data by Brady et al. ${ }^{15}$ that indicate a mild increase in $\tau_{\text {coll }}$ upon increasing $b$ at fixed $v_{p}$. Noticing that the fraction of postcollisional complexes dissociating within 40 ps from the collision decreases upon decreasing $b$, it would seem reasonable to attribute the presence of such fast dissociating species to the ballistic dynamics responsible for the nonstatistical branching ratios previously discussed, an idea substantiated by a direct visual inspection of trajectories evolution. Conversely, the difference with the work of Brady et al. may be due to the smaller and colder $\left(E_{\mathrm{int}}=0\right.$ hartree $)$ clusters used and to a slow conversion of rotational kinetic energy into vibrational one. ${ }^{17}$

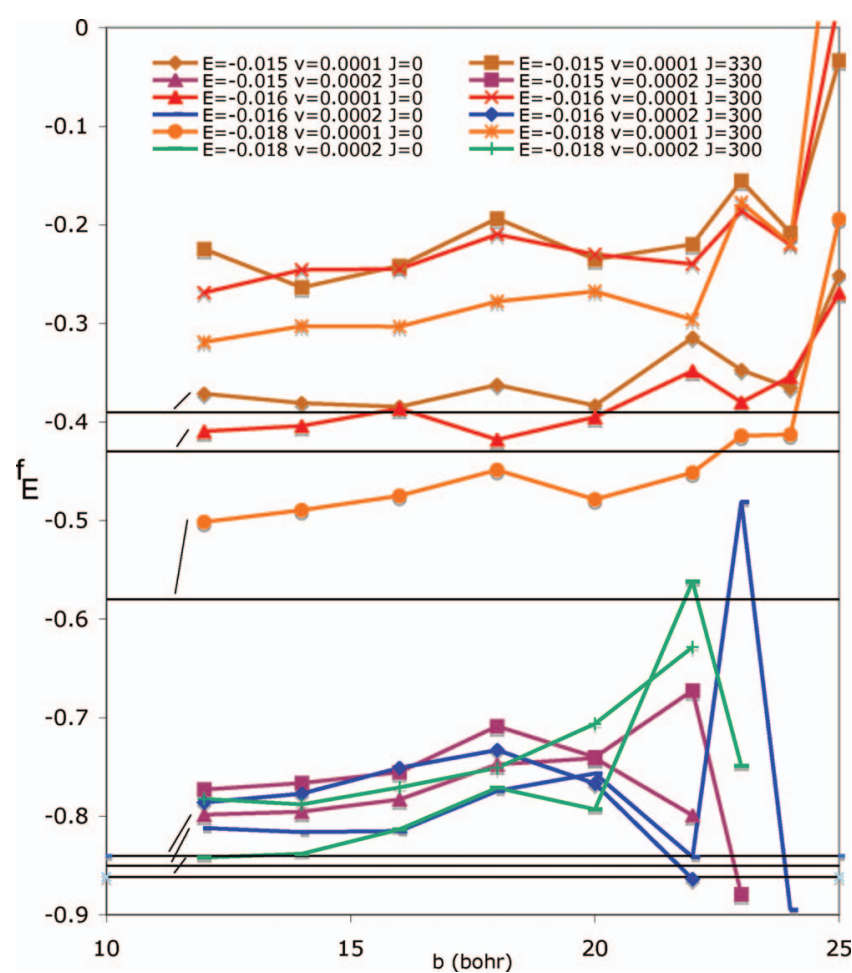

FIG. 6. Fraction $f_{E}$ of the projectile kinetic energy transferred to the dissociating monomer after projectile capture by $\mathrm{LJ}_{19}$ as a function of the impact parameter $b$ for twelve $\left(E, v_{p}, J\right)$ cases, with $E=-0.018,-0.016$, and -0.015 hartree, $J=0$ and $J=J_{\max }=300$ a.u., and $v_{p}=0.0001,0.0002$ a.u. (see caption Fig. 1 for conversion)/Results obtained using 1000 trajectories at the indicated values of $E, b$, and $v_{p}$ and $b$. The horizontal lines indicate the average value of $f_{E}$ obtained simulating the dissociation of an ensemble of $\mathrm{LJ}_{20}^{*}$, with statistically distributed initial conditions satisfying the value of total energy and angular momentum for collisions happening between a nonrotating cluster and a projectile aimed at $b=12$ (indicated by thin black lines). Standard errors are roughly $5 \%-10 \%$ of the shown $f_{E}$ values for $b$ $<20 \mathrm{bohr}$; at larger values of the impact parameter, standard errors and $f_{E}$ have similar magnitude due to the small number of capture events.

As an additional consequence of the aforementioned deviations from Rice-Ramsperberg-Kassel-like ${ }^{23}$ behavior, the postcollision lifetime $\tau_{\text {coll }}$ would be expected to decrease upon increasing $b$ due to a decrease in the $P_{s} / P_{c}$ ratio, which implies a reduced energy exchange. Such behavior is clearly present in high speed collisions as shown in panel b of Fig. 4 , and also provides a simpler explanation for the faster short time postcollisional decay found in Ref. 17 when $b>0$ than invoking the proneness to decompose of a just formed complex.

As a final step in our study of the postcapture dynamics of $\mathrm{LJ}_{n}$ with $\mathrm{LJ}$ projectiles, we investigated the fractional amount of energy exchanged $f_{E}=\Delta E / \epsilon_{p}=\left(\epsilon-\epsilon_{p}\right) / \epsilon_{p}$ during the process $\mathrm{LJ}_{n}^{\prime}+\mathrm{LJ}^{\prime} \rightarrow \mathrm{LJ}_{n+1}^{*} \rightarrow \mathrm{LJ}_{n}^{\prime \prime}+\mathrm{LJ}^{\prime \prime}$. In the previous equations, $\Delta E$ represent the difference in kinetic energy between $\mathrm{LJ}^{\prime \prime}$ and $\mathrm{LJ}^{\prime}, \epsilon_{p}=m v_{p}^{2} / 2$ and $\epsilon$ are the kinetic energy of the incoming and departing monomers, and ' and "indicate possible differences in energy content between product and reactant species. Figure 6 shows the $\mathrm{LJ}_{19}$ results for all the values of $\epsilon_{p} / E$ used previously, and $J=0$ and $J=J_{\max }$ $=300 \hbar$. Similarly to what was previously discussed for other quantities, the dependence of $f_{E}$ with respect to $b$ appears to be very weak (within the statistical fluctuations of our re- 
sults), quite likely due to fast energy redistribution between vibrational modes and a small rotational energy content. ${ }^{17}$ In other words, it appears that a single "oscillation" of the projectile in the attractive well of the cluster is sufficient to almost completely redistribute the collision energy, and that the slight increase in $f_{E}$ upon increasing $b$ may be ascribed to the propensity of a cluster with $J>0$ to emit a slightly faster monomer [i.e., with a higher kinetic energy release (KER)] as discussed in Ref. 24. A similar argument based on the KER can also be used to explain the clear evidence that, at low $v_{p}$, an emitted monomer departs with a higher fraction of the projectile kinetic energy the higher $J$ (e.g., see $J=300 \hbar$ cases in Fig. 6) as a consequence of an increased contribution of the relative rotational energy component to the KER at high $J .^{24}$ Albeit partially masked by the higher $\epsilon_{p}$ value, this finding is also true at high $v_{p}$. Notice, however, that the absolute magnitude of such contribution is very low compared with the internal energy of the clusters, so that the effect of the higher kinetic energy injected in the emitted particle on the physical properties of the remaining aggregate is expected to be small. ${ }^{25}$

From Fig. 6, it is also apparent that a larger fraction of $\epsilon_{p}$ is left in the cluster upon dissociation in the case of high speed collisions. This indicates a stronger propensity for the dissociating moiety to leave with far less kinetic energy $\epsilon$ than originally possessed by the projectile the higher $\epsilon_{p}$, a fact that it is connected to the steep increase in the density of states of atomic clusters upon increasing their vibrational energy. In fact, the principle of equal a priori probability indicates that the most probable energy distribution for the dissociated clusters is the one that maximizes the product $\omega\left(E_{t}-\epsilon_{0}-\epsilon\right) \epsilon$, where $\omega$ is the density of states of $\mathrm{LJ}_{n}$, the multiplicative term $\epsilon$ is proportional to the density of states of the dissociated moiety, while $E_{t}$ and $\epsilon_{0}$ are, respectively, the total energy of $L J_{n+1}^{*}$ and the monomer dissociation energy. Because $\omega\left(E_{t}-\epsilon_{0}-\epsilon\right)=\omega\left(E+\epsilon_{p}-\epsilon\right)$ is expected to decrease faster upon increasing $\epsilon$ the higher $\epsilon_{p}$ is [i.e., $\partial \omega(E$ $\left.+\epsilon_{p}-\epsilon\right) / \partial \epsilon$ is more negative the higher $\epsilon_{p}$ ], the maximum of $\omega \epsilon$ will be located at lower values of $\epsilon$ the higher is $\epsilon_{p}$ favoring more the dispersion of the initial kinetic energy in the clusters. As a quantitative example, we mention that, assuming an harmonic behavior for the $s=3 n-6$ vibrations of $\mathrm{LJ}_{n}$, one obtains $f_{E}=E / s \epsilon_{p}-(s-1) / s$; given that $\epsilon_{p}, E>0$, and $s>1$, this clearly indicates that $f_{E}$ should be expected to decrease upon increasing $\epsilon_{p}$.

Albeit the behavior of $f_{E}$ versus $b, E$, and $J$ is perfectly in line with what would be expected following a fast energy redistribution, a direct confirmation of the statistical energy distribution in postcapture dissociating clusters can only be obtained comparing directly with the prediction for $f_{E}$ obtained using trajectories with initial conditions sampled accordingly to the statistical distribution associated to the chosen $(E, J, b)$ set (i.e., an energy of $E+\epsilon_{p}$ and a total angular momentum of $\left.J+m b \times v_{p}\right)$. As shown in Fig. 6, such results are in good agreement with the ones obtained following the postcapture dissociation, differing from each other by limited amounts. Notice that the agreement between dynamically and statistically computed $f_{E}$ may be improved by selecting higher $N_{\text {tp }}$ values.

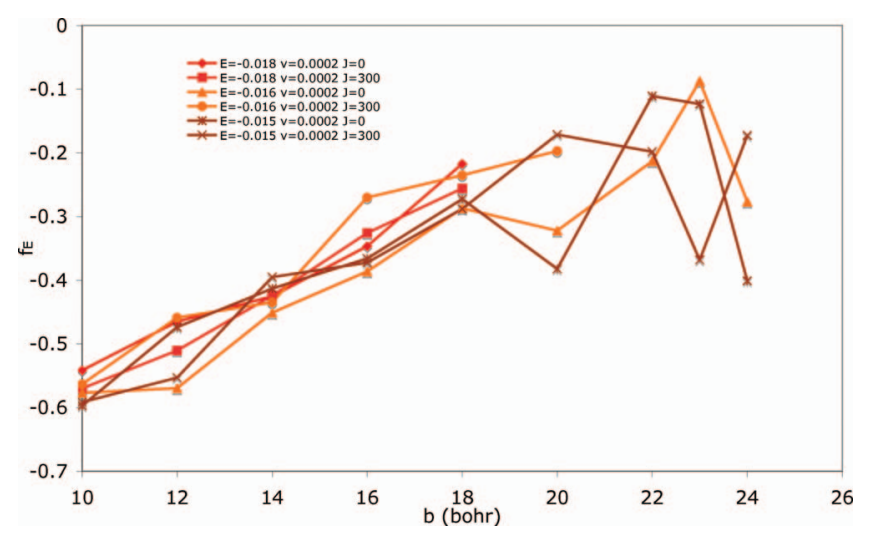

FIG. 7. Fraction $f_{E}$ of the projectile kinetic energy remaining in the projectile after the latter has collided without being captured by $\mathrm{LJ}_{19}$ as a function of the impact parameter $b$ for six $\left(E, v_{p}, J\right)$ cases $(E=-0.018,-0.016$, and -0.015 hartree, $J=0$ and $J=J_{\max }=300$ a.u., and $v_{p}=0.0002$ a.u., see caption Fig. 1 for conversion). Standard errors are roughly $5 \%-10 \%$ of the shown $f_{E}$ values for $b<20$ bohr; at larger values of the impact parameter, standard errors and $f_{E}$ have similar magnitude due to the small number of collision events. Results obtained using 1000 trajectories at the indicated values of $E, b$, and $v_{p}$ and $b$.

According to what just discussed, our definition of captured monomer appears to guarantee enough time to $\mathrm{LJ}_{n+1}^{*}$ to largely redistribute the kinetic energy carried in the system by the projectile upon collision. Thus, given the high fraction $(>90 \%)$ of captured monomer during low $v_{p}$ collisions, one would therefore conclude that collision and capture are almost indistinguishable for all practical purposes in these conditions. Conversely, it would be useful to gather information on the energy transfer in the case of trajectories producing a collision but not a capture, which are likely to happen when the projectile has a higher kinetic energy and is aimed at the cluster boundary. To this end, we computed the average $f_{E}$ for trajectories with high $v_{p}$ as a function of the impact parameter $b$. As one would expect, the results shown in Fig. 7 indicate that a simple collision transfers less energy from the projectile to the aggregate than a capture (roughly $70 \%$ at $b=10$ and rapidly decreasing with $b$ ), but also that $f_{E}$ decreases upon increasing $b$ much faster than expected on the basis of a possible contribution due to the rotational energy component. This can be explained considering that the probability of a "head to head" hit between projectile and monomers in $\mathrm{LJ}_{n}$ decreases upon increasing $b$, leading to a higher fraction of glancing collisions whose kinematics disfavors energy transfer.

\section{CONCLUSIONS}

Seeking for possible simplifications in the description of a nucleating vapor by means of a microcanonical theory, in this study we have investigated the dependence of several key quantities $\left(P_{c}, P_{s}, \tau_{\mathrm{dyn}}\right.$, and $\left.f_{E}\right)$ with respect to collision variables such as the impact parameter $b$, the target angular momentum $J$ and internal energy $E$, and the speed of the projectile $v_{p}$. In general terms, our numerical investigation indicates that postcapture $\mathrm{LJ}_{n+1}^{*}$ closely follow a statistical behavior [Figs. 3(a), 5(a), and 6] regardlessly of the energy 
content of the $\mathrm{LJ}_{n}$ clusters, their angular momentum and the initial orbital momentum $L=m v_{p} \times b$ of the composite projectile-target system thanks to fast energy exchange. ${ }^{17}$ In turn, this supports the possibility of using postcapture energized species as intermediates in the description of a supersaturated noble gas vapor. Moreover, our results have also highlighted a weak sensitivity of $P_{s}$ [Fig. 1(b)] with respect to $J$ and $E$, and of $\tau_{\text {dyn }}$ [Fig. 4(a)] with respect to $J$ and $b$, which can be explained by the presence of small structural changes in the targets as a function of $E$ and $J$ (Fig. 2) and by a limited influence of the rotational energy on the density of states of $\mathrm{LJ}_{n+1}^{*}$.

Similar conclusions hold only partially when high $v_{p}$ results for $\tau_{\text {coll }}$ are considered [Fig. 4(b)], which decreases upon increasing $b$. We attribute this finding to the presence of nonstatistical behavior caused by a residual tangential component of $v_{p}$ after a collision has taken place. Notice, however, that the postcollisional species responsible for the decrease in $\tau_{\text {coll }}$ should not be considered as properly formed $\mathrm{LJ}_{n+1}^{*}$ intermediates (they do not satisfy our dynamical criterium for capture), but only as transient entities whose impact on the nucleation process is to provide a means for energy transfer between monomers and clusters with reasonably long lifetime to allow a statistical distribution of their internal energy. From our data one would estimate that less than $20 \%$ of the total collisions at high $v_{p}$ (Ref. 26) could produce nonstatistically equilibrated $\mathrm{LJ}_{n+1}^{*}$, a fraction of the total events that appears likely to have only a minor impact on the total energy transfer. ${ }^{27}$ Even so, one may wish to include such contribution to the global energy transfer, a task that is expected to require a dedicated treatment (e.g., see Ref. 28). In doing this, the dynamical criterium differentiating capture from collision employed in this study seems to provide a convenient approach for sifting the two different classes of events and allowing one to focus only on simple collisions when it comes to the process of parameterizing energy exchange kernels,

As suggested in the Introduction, the weak or completely lacking sensitivity of $P_{s}$ on $E$ and $J$, and of $\tau_{\text {dyn }}$ on $J$ and $b$ can be exploited to greatly simplify the set of MEs (Ref. 4) that one wishes to employ in describing the formation of clusters from a supersaturated vapor. Physically speaking, they allow one to more confidently assume that only $n$ and $E$ play an important role in defining the property of a nucleating cluster, a possibility previously suggested in Ref. 10 and employed by Barrett. ${ }^{11}$ Notice, however, that this assumption is conditional to the fact that no cluster with extremely high angular momentum are formed during the nucleation, i.e., that the clusters are formed so that $J \approx J_{\max }$ at most. In the case $J \gg J_{\max }$, the centrifugal distortion ought to be taken in consideration, but the existence of a large population of such species appears highly unlikely given the necessity of capturing a very fast monomer aimed at the aggregate boundary to generate a large $J$ in a single collision [see Fig. 1(b) for a quantitative estimate of such possibility]. The assumption of no dependence on $J$, instead, allows one to integrate over it both sides of the integro-differential equations defining the time derivative of $N(n, E, J)$, the population of $\mathrm{LJ}_{n}$ clusters with energy and angular momentum $J$, in terms of dissociation and capture processes. ${ }^{4}$ In turn, one is allowed to introduce integral quantities such as $N(n, E)=\int N(n, E, J) d J$ and $J$-independent rate constants, the latter neglecting the dependency on the initial and final value of cluster angular momentum, with the important consequences of reducing largely the total cost for obtaining the data sets of both $k_{d}(n)$ and $k_{c}(n)$ needed for the MEs.

On a similar vein, we conclude mentioning that the smooth and regular behavior of $P_{s}$ with respect to $E$ and $b$ also suggests that it may be possible to further reduce the computational effort in estimating $k_{c}$. Such an estimate may be obtained employing simplificated approaches based, for instance, on $E$-dependent average interaction potentials and simple models for the postcollision energy exchange needed to transform a collision into capture.

\section{ACKNOWLEDGMENTS}

The author acknowledges useful comments from Gabriele Morosi and Emanuele Curotto.

${ }^{1}$ J. Wedekind and D. Reguera, J. Chem. Phys. 127, 154516 (2007).

${ }^{2}$ H. Matsubara, T. Koishi, T. Ebisuzaki, and K. Yasuoka, J. Chem. Phys. 127, 214507 (2007).

${ }^{3}$ S. Toxvaerd, J. Chem. Phys. 115, 8913 (2001).

${ }^{4}$ P. Schaaf, B. Senger, J.-C. Voegel, R. K. Bowles, and H. Reiss, J. Chem. Phys. 114, 8091 (2001).

${ }^{5}$ K. K. Tanaka, K. Kawamura, H. Tanaka, and K. Nakazawa, J. Chem. Phys. 122, 184514 (2005).

${ }^{6}$ P. R. ten Wolde and D. Frenkel, J. Chem. Phys. 109, 9901 (1998).

${ }^{7}$ J. Merikanto, E. Zapadinsky, A. Lauri, and H. Vehkamäki, Phys. Rev. Lett. 98, 145702 (2007).

${ }^{8}$ G. K. Schenter, S. M. Kathmann, and B. C. Garrett, J. Chem. Phys. 110, 7951 (1999).

${ }^{9}$ J. C. Barrett, J. Chem. Phys. 128, 164519 (2008).

${ }^{10}$ M. Mella, J. Chem. Phys. 130, 084108 (2009).

${ }^{11}$ J. C. Barrett, J. Chem. Phys. 126, 074312 (2007)

${ }^{12}$ S. Weerasinghe and F. G. Amar, J. Chem. Phys. 98, 4967 (1993).

${ }^{13}$ G. H. Peslherbe and W. L. Hase, J. Chem. Phys. 105, 7432 (1996).

${ }^{14}$ J. W. Brady, J. D. Doll, and D. L. Thompson, J. Chem. Phys. 74, 1024 (1981).

${ }^{15}$ J. W. Brady, J. D. Doll, and D. L. Thompson, J. Chem. Phys. 71, 2467 (1979).

${ }^{16}$ I. Napari, H. Vehkamäki, and K. Laasonen, J. Chem. Phys. 120, 165 (2004).

${ }^{17}$ I. Napari and H. Vehkamäki, J. Chem. Phys. 124, 024303 (2006)

${ }^{18}$ H. W. Schranz, L. M. Raff, and D. L. Thompson, J. Chem. Phys. 94, 4219 (1991).

${ }^{19}$ D. L. Bunker and W. L. Hase, J. Chem. Phys. 59, 4621 (1973).

${ }^{20}$ F. H. Stillinger, J. Chem. Phys. 38, 1486 (1963).

${ }^{21}$ Z. H. Li and D. G. Truhlar, J. Phys. Chem. C 112, 11109 (2008)

${ }^{22}$ See EPAPS supplementary material at http://dx.doi.org/10.1063/ 1.3239476 for $P_{c}$ and $\tau_{\text {coll }}$ computed for $\mathrm{LJ}_{8,14,19}$ and $P_{s}, \tau_{\text {dyn }}$ and $\Delta E$ for $\mathrm{LJ}_{8,14}$.

${ }^{23}$ T. Baer and W. L. Hase, Unimolecular Reaction Dynamics (Oxford University Press, Oxford, 1996).

${ }^{24}$ F. Calvo and P. Parneix, J. Chem. Phys. 119, 256 (2003).

${ }^{25}$ F. Calvo and P. Parneix, J. Chem. Phys. 126, 034309 (2007).

${ }^{26}$ Our estimate for the fraction of high $v_{p}$ collision that could lead to nonstatistical dynamics was computed as the fraction of trajectories in the range of $b$, where $P_{c}$ drops with the proper $b$-weighting; as such it is likely to be an upper bound to the real value since it does not take into account the trajectories that are captured in the same range of $b$ [see Fig. 1(b)]. A total estimate for the possible effect of nonstatistical dynamics 
over all possible velocities would require to carry out a large number of trajectories with thermalized projectiles in order to compute the appropriate ensemble average at $120 \mathrm{~K}$ and it is considered at the present outside the scope of this work. As a rough estimate, however, one might consider that high speed collision (say $v_{p}>0.0018$ a.u.) are less than
$20 \%$ of the total collisions, with only $1 / 5$ of these (i.e. only $5 \%$ of the total) being likely to show nonstatistical dynamics.

${ }^{27}$ L. Ming, J. Davidsson, and S. Nordholm, Chem. Phys. 201, 121 (1995).

${ }^{28}$ L. E. B. Börjesson, L. Ming, and S. Nordholm, Chem. Phys. 221, 253 (1997). 